\title{
Les enjeux identitaires de l'écriture de l'histoire dans le Yémen contemporain
}

Identity Issues in Writing the History of Contemporary Yemen

Jean Lambert

\section{(2) OpenEdition}

1 Journals

Édition électronique

URL : https://journals.openedition.org/transcontinentales/411

DOI : 10.4000/transcontinentales.411

ISBN : 978-2-7351-1561-7

ISSN : $1775-397 X$

\section{Éditeur}

Editions de la maison des sciences de l'homme

\section{Édition imprimée}

Date de publication : 30 juin 2008

Pagination : $57-71$

ISBN : 978-2-200-92511-6

ISSN : $1950-1684$

\section{Référence électronique}

Jean Lambert, «Les enjeux identitaires de l'écriture de l'histoire dans le Yémen contemporain »,

Transcontinentales [En ligne], 6 | 2008, document 4, mis en ligne le 06 avril 2011, consulté le 21

septembre 2021. URL : http://journals.openedition.org/transcontinentales/411 ; DOI : https://doi.org/ 10.4000/transcontinentales. 411 


\title{
Les enjeux identitaires de l'écriture de l'histoire dans le Yémen contemporain
}

\author{
Jean Lambert
}

Au Yémen, l'émergence d'une nation a des bases plus solides que dans d'autres pays de la région dont les frontières ont été tracées entièrement par la colonisation : au cours des trois derniers millénaires, il s'est succédé dans ce coin sud-ouest de la péninsule Arabique des entités politiques se distinguant par une civilisation écrite, par des langues ou des dialectes locaux spécifiques, par des écoles religieuses hétérodoxes et par des pouvoirs politiques plus ou moins indépendants des grands empires, en particulier, pendant la période islamique, de l'Empire abbasside et de l'Empire ottoman ${ }^{1}$. Mais ce n'est que progressivement que certaines entités, qui ne recouvrent pas nécessairement l'ensemble du territoire délimité par les frontières actuelles, ont fini par être désignées du nom de "Yémen » ${ }^{2}$. Cette lente émergence d'une entité nationale yéménite a donc souvent pris des chemins de traverse, y compris à l'époque actuelle. Dans un pays qui a été divisé en deux depuis le $\mathrm{XIX}^{\mathrm{e}}$ siècle - avec au Nord, la constitution d'un État théocratique au début $\mathrm{du} \mathrm{xx}^{\mathrm{e}}$ siècle à l'issue d'une guerre d'indépendance contre les Ottomans, puis une révolution républicaine (la République arabe du Yémen) en 1962, et au Sud, une occupation britannique longue de cent vingt ans qui se conclut par l'établissement d'un État marxiste (la République démocratique et populaire du Yémen) en 1967 -, l'unité yéménite finalement proclamée en 1990 à la faveur de l'effondrement du bloc soviétique ne s'est concrétisée vraiment qu'après une guerre civile en 1994, et reste jusqu'à maintenant problématique.

1 - Michel Tuchscherer, «Introduction», Revue des mondes musulmans et de la Méditerranée, $\mathrm{n}^{\circ} 67$ Le Yémen, passé et présent de l'unité, 1993.

2 - Franck Mermier, "Présentation», Revue des mondes musulmans et de la Méditerranée, $\mathrm{n}^{\circ} 121$ 122: Yémen Territoires et identités, avril 2008, p.9-16 (en ligne: http://remmm.revues.org/document4703.html). 
L'histoire du Yémen est donc particulièrement complexe, tant avant l'islam qu'après son apparition, tant aux époques anciennes qu'à l'époque contemporaine. Et de nombreux aspects sociologiques et politiques de cette histoire ont des prolongements dans la société actuelle. Par ailleurs, cette histoire du Yémen est encore loin d'être écrite.

Abstraction faite des difficultés d'accès aux vestiges archéologiques et aux documents écrits, rendre compte de cette histoire, la mettre par écrit et en tirer, éventuellement, des enseignements pour aujourd'hui est en soi une tâche ardue. En général, on trouve chez les divers auteurs différentes versions de certains événements clés, en particulier selon l'origine régionale de ces auteurs. En outre, les historiens professionnels n'ont pas toujours acquis la légitimité nécessaire pour produire des versions autorisées, et ce sont souvent des non-spécialistes qui se font le plus entendre. La première Histoire générale du Yémen ${ }^{3}$ n'a été publiée à Sanaa que récemment, et elle est encore peu soucieuse des critères académiques modernes ${ }^{4}$. Dès lors, il est difficile de distinguer des vérités historiques indiscutables parmi les différentes mémoires communautaires ${ }^{5}$. Ces différentes versions de l'histoire yéménite pourraient être analysées comme Ahmed Beydoun l'a fait pour l'histoire libanaise ${ }^{6}$, c'est-à-dire en les confrontant les unes aux autres, en essayant de comprendre leurs motivations politiques profondes, mais sans grand espoir de parvenir à une vérité historique absolue.

Compte tenu des continuités historiques qui sont indéniables au Yémen, on ne peut pas abstraire l'écriture de l'histoire du contexte de l'État-nation, de ses modèles dominants et des enjeux de sa construction, jusque dans sa contestation. Il s'agira alors de distinguer des versions alternatives à la version officielle (des counter-narratives) ${ }^{7}$ permettant de mettre en question les versions nationalistes de l'histoire ${ }^{8}$. Pour le cas du Yémen, se pose également la question importante de l'émergence de traits

3 - Mohammed Yahyâ al-Haddâd, Histoire générale du Yémen. I. Avant l'islam; II. Dans le cortège de l'islam; III. Le Yémen moderne et contemporain, Sanaa, Ministère de la Culture et du Tourisme, 2004 (en arabe).

4 - L'ouvrage suit un découpage chronologique classique (Antiquité, islam, époque moderne, période contemporaine), mais recourt assez peu à des sources de première main.

5 - Au Yémen, "[l]a mémoire collective a plus affaire au refoulement et à l'occultation qu'à une légende des siècles parcourue de figures tutélaires incarnant le destin national ", écrit Franck Mermier («Les héritages d'une histoire morcelée», in Rémy Leveau, Franck Mermier et Udo Steinbach (dir.), Le Yémen contemporain, Paris, Karthala, 1999, p. 33). Le même auteur note également la nécessité d'une «réappropriation collective du passé».

6 - Ahmed Beydoun, Identité confessionnelle et temps social chez les historiens libanais contemporains, Beyrouth, Université libanaise, 1984.

7 - Pour le Yémen et la péninsule Arabique, voir Madawi al-Rasheed et Robert Vitalis (Counternarratives: History, Contemporary Society and Politics in Saudi Arabia and Yemen, New York, Palgrave Macmillan, 2003), et notamment l'article de Sheila Carapico : "Arabia Incognita. An Invitation to Arabian Peninsula».

8 - Certaines études de "counter-narratives" tendent à contester que la nation soit une communauté. Ainsi, pour Prasenjit Duara, "[a]lors qu'il a été montré que la nation est une relation instable et contingente», l'histoire est souvent convoquée pour «renforcer la mystique de la nation" et "sa prétention douteuse à une subjectivité en évolution" (P. Duara, Rescuing History from the Nation : Questioning Narratives of Modern China, Chicago, University of Chicago Press, 1995). Cependant, cette approche stimulante ne semble pas en mesure d'invalider la force "imaginaire» et symbolique de la construction des États-nations telle que l'a analysée par exemple Benedict Anderson (Imagined Communities : Reflections on the Origin and Spread of Nationalism, Londres et New York, Verso, 1991). 
précurseurs d'une nation avant la période contemporaine (bien avant l'influence du nationalisme moderne), prémices qui ne sont pas négligeables?

Il faudra également tenir compte d'un autre paramètre lié à la mondialisation du savoir. Les chercheurs étrangers étant très impliqués dans l'étude de l'islam médiéval au Yémen, ils sont parfois amenés à produire une version de certains événements de l'histoire du pays qui diverge de la version officielle, et à prendre ainsi parti, bien involontairement, dans ces débats contemporains. Il sera donc autant question de la réception des travaux historiques par la société et le grand public, que de ces travaux eux-mêmes. Contrairement à ceux d'Umberto Eco, les «lector in fabula» yéménites (lettrés traditionnels, intellectuels modernistes et vulgarisateurs en tous genres) ne possèdent pas nécessairement les codes qui sont ceux de la science moderne, largement occidentale; aussi construisent-ils volontiers leurs propres versions des données mises au jour par les archéologues et les historiens - des versions non autorisées dans la communauté scientifique internationale, mais très influentes localement. C'est donc d'une expérience très particulière dont je voudrais parler ici, qui fait appel à une anthropologie politique comprenant nécessairement une certaine part d'altérité culturelle...

\section{Un passé pré-islamique valorisé}

Depuis au moins quarante ans, il est notable que les Yéménites valorisent particulièrement leur passé pré-islamique. Cette attitude, dans un pays arabe et musulman, n'est pas si courante, si l'on compare par exemple avec leurs voisins d'Arabie saoudite, du Golfe, d'Égypte ou même du Maghreb. La reine de Sabâ ${ }^{10}$, le barrage de Mareb ${ }^{11}$ et un certain nombre de héros guerriers pré-islamiques comme As'ad al-Kâmil ${ }^{12}$, Sayf ben Dhî Yazen ${ }^{13}$ et bien d'autres, sont particulièrement valorisés en public, en particulier dans les manuels d'histoire et par différents discours nationalistes contemporains faisant une grande place à la légende $e^{14}$.

Simultanément, cette approche légendaire de l'histoire pré-islamique du Yémen commence à être battue en brèche par l'émergence d'une nouvelle génération d'archéologues yéménites qui apportent eux-mêmes par leurs découvertes et dans

9 - Voir dans la Revue des mondes musulmans et de la Méditerranée, le numéro 67 : Yémen, passé et present de l'unité, dirigé par Michel Tuchscherer (1993), et le numéro 121-122: Yémen Territoires et identités (avril 2008), notamment l'article de Franck Mermier, déjà cite, et la contribution de Laurent Bonnefoy sur «Les identités religieuses contemporaines au Yémen» (p. 199-213).

10 - Reine légendaire figurant dans la Bible et le Coran, n'ayant pas d'existence historique attestée, mais faisant référence au royaume historique de Sabâ ( $\mathrm{VIII}^{\mathrm{e}}$ siècle av. J.-C. - I ${ }^{\mathrm{er}}$ siècle apr. J.-C.).

11 - Barrage monumental ayant fait la fortune agricole du royaume de Sabâ, entre le vIII ${ }^{e}$ siècle avant J.-C. et le $\mathrm{vI}^{\mathrm{e}}$ siècle après J.-C.

12 - Un des rois himyarites, unificateur du Yémen antique au Ive siècle, sans doute le premier à avoir adhéré au monothéisme.

13 - Héros sudarabique du vi ${ }^{e}$ siècle qui aurait chassé les Abyssins du pays.

14 - Il y a plusieurs années, j'avais décrit comment le mythe de la reine de Sabâ et du barrage de Mareb était un enjeu d'interprétation pour différentes composantes sociopolitiques du Yémen dans les années 1990 (J. Lambert "Sabâ et le barrage de Mareb. Récit fondateur et temps imaginé», Peuples de la Méditerranée, $\mathrm{n}^{\circ}$ 56-57, juillet-décembre 1991, p. 141-176). Depuis cette date, cette tendance n'a cessé de se confirmer. L'intérêt du Yémen pour son passé pré-islamique rappelle par de nombreux aspects la relation qu'entretient l'Égypte avec son passé pharaonique. 
leurs écrits une version plus proche des données du terrain. Mais «cette nouvelle version a encore du mal [...] à s'affirmer auprès de la population ${ }^{15}$ ». On peut néanmoins remarquer que les approches légendaires se nourrissent à leur tour des dernières découvertes en les réinterprétant à leur façon ${ }^{16}$.

Le fait que certaines de ces légendes soient mentionnées dans le Coran les distingue; elles instituent ainsi une filiation prestigieuse et proprement yéménite avec l'islam ${ }^{17}$. Avant l'islam, cette filiation alternative faisait écho à une concurrence politique et spirituelle qui existait déjà entre le nord et le sud de l'Arabie, et plus particulièrement entre la Kaaba de La Mekke et la cathédrale chrétienne de Sanaa, comme le montre l'épisode de l'attaque du roi Abraha contre La Mekke en $552^{18}$, connue dans le Coran comme "la campagne de l'Éléphant», sourate 105. Cette opposition entre nord et sud de l'Arabie est importante pour l'identité historique du Yémen ${ }^{19}$, et elle a connu bien d'autres développements qui ont permis aux Yéménites de contourner la filiation qoraychite ${ }^{20}$ située hors de leur sphère géo-culturelle.

Le cour du problème que nous allons examiner se situe dans l'histoire islamique du Yémen, dont les prolongements contemporains ont des implications politiques plus perceptibles.

\section{Mille ans de domination politique et intellectuelle du zaydisme au Yémen}

Pendant près de mille ans, le Yémen fut dominé par une entité politique bien particulière, l'imamat zaydite ${ }^{21}$. C'était un régime théocratique fondé sur une secte

15 - Mounir Arbach et Rémy Crassard, «L'Arabie du Sud vue de l'intérieur. Recherches archéologiques menées par les Yéménites», Chroniques yéménites, n 14, 2007, p. 10.

16 - Ainsi, sur des simples présomptions et un rapprochement linguistique douteux avec la chronologie babylonienne, l'« historien» Mohammed al-Farah fait remonter la chronologie sabéenne à une période plus ancienne (1500 ans av. J.-C.) que celle reconnue par la communauté des archéologues (1000 ans av. J.-C. environ) (Farah, Les tubbâ' sabéens du Yémen, Sharja, Dâr al-Thaqâfa al-'Arabiyya, 2002, p. 11-12). Ailleurs, le même auteur fait remonter la civilisation sudarabique à 9000 ans avant J.-C., en jouant sur la confusion entre la datation de l'âge de bronze au Yémen et la datation des débuts de la période historique (Farah, Découvertes récentes sur l'histoire de l'État et de la civilisation de Sabâ, Sanaa, Ministère de la Culture, 2004, p. 5-6). L'ancienneté est à l'évidence recherchée comme source de prestige...

17 - Et au-delà, avec le monothéisme et les périodes plus anciennes de la Bible : selon la légende, rapportée entre autres par l'historien yéménite al-Hamdânî, Sanaa aurait été fondée par Sem, le fils de Noé. Comme dans beaucoup d'autres points du monde musulman, les restes de l'arche de Noé sont activement recherchés, en combinant une numérologie mystique du Coran et... les coordonnées géographiques de Google Earth ! La région de Sanaa se prête à ce genre d'élaboration imaginaire, dans la mesure où son site naturel en cuvette aurait pu favoriser l'accumulation des eaux du Déluge.

18 - Joëlle Beaucamp, Françoise Briquel-Chatonnet et Christian Robin, «La persécution des chrétiens de Najrân et la chronologie himyarite», ARAM, $\mathrm{n}^{\circ} 11-12,1999-2000$ (paru en 2001), p. 73.

19 - "Abraha se comporte comme le successeur des grands souverains himyarites du ve siècle» (J. Beaucamp, F. Briquel-Chatonnet et Ch. Robin, art. cit., 1999-2000, p. 78).

20 - La tribu de Qoraysh, installée à La Mekke avant l'islam, était celle du Prophète, mais du vivant de ce dernier, elle l'avait combattu (ce que les musulmans yéménites ne se font pas faute de lui reprocher).

21 - Cet État zaydite eut la plupart du temps une stabilité limitée, comme le montrent les fréquents changements de capitale (Michel Tuchscherer, art. cit., 1993, p. 6). 
hétérodoxe de l'islam ${ }^{22}$. Le premier imam, al-Hâdî, avait pris pied à Saada, dans le nord du Yémen, en $898^{23}$. Pendant près de dix siècles, un État zaydite se maintint tant bien que mal dans la zone des hauts plateaux du Yémen ${ }^{24}$. Ce pouvoir fut renversé en 1962 au Yémen du Nord par une révolution républicaine. À partir de 1990, l'ancien Yémen du Nord fut unifié à l'ancien Yémen du Sud, constituant ainsi un nouvel État, la république du Yémen. Or, malgré ces bouleversements considérables, on observe des continuités remarquables : jusqu'à aujourd'hui, la région des hauts plateaux, qui entoure la capitale, Sanaa, reste la principale source du pouvoir, avec notamment plusieurs tribus alliées appartenant à la confédération Hâshed, dont le grand sheykh était 'Abdallah al-Ahmar ${ }^{25}$, un personnage clé du régime yéménite ${ }^{26}$. Cela pose la question de la pérennité de l'exercice du pouvoir par la communauté zaydite, et au-delà, de la possible existence transhistorique d'une telle communauté, de sa nature et de son identitée ${ }^{27}$.

Pendant cette période de domination zaydite, seuls les membres de la famille des Hachémites ${ }^{28}$, les sayyid, pouvaient détenir le pouvoir suprême. Sur le plan sociologique, les sayyid formaient jusqu'à maintenant une aristocratie religieuse et intellectuelle, une classe sociale très consciente d'elle-même et qui se distinguait par un ensemble de traits et de marqueurs identitaires, en particulier l'endogamie et l'hypergamie ${ }^{29}$. L'une des principales spécificités du zaydisme historique était une théorie du pouvoir et de l'imamat, selon les principales conditions qui avaient été posées par son fondateur, al-Hâdî, ou par certains successeurs directs : 1) le pouvoir suprême (l'imamat) devait être détenu uniquement par un Hachémite; 2) si le pouvoir n'était pas exercé selon les lois de l'islam, n'importe quel membre lettré de la Famille pouvait prendre les armes contre un souverain injuste et le détrôner. Les sayyid étaient donc étroitement liés à cette théorie du pouvoir et à sa place dans la définition même du zaydisme. Inversement, la nature du zaydisme est étroitement liée à cette question qui se pose nécessairement aux intellectuels d'origine zaydite : le zaydisme peut-il exister en tant que tel sans imam? Comment

22 - L'origine du zaydisme remonte à Zayd ben 'Alî, le quatrième imam de la tradition chiite, mort en 740 sous la répression ommeyade. Très influencés par le rationalisme de la pensée mo'tazilite, les zaydites se distinguent des autres courants chiites, septimains et duodécimains, par une plus grande proximité à l'islam sunnite majoritaire.

23 - Venant du Tabaristan où avait existé précédemment un petit État zaydite, les partisans d'alHâdî pourront être ultérieurement considérés comme doublement étrangers : comme Arabes du Nord et comme Persans.

24 - Son extension progressive à tout le haut plateau yéménite a connu plusieurs phases qui ont laissé des strates dans la société contemporaine (Gochenhour, «Towards a Sociology of the Islamization of Yemen", in B.R. Pridham, Contemporary Yemen, Politics and Historical Background, Londres, Croom Helm, University of Exeter, 1984).

25 - Le sheykh 'Abdallah al-Ahmar est disparu en décembre 2007.

26 - Franck Mermier, «Les héritages d'une histoire morcelée», art. cit., 1999, p. 19.

27 - Samy Dorlian, «Zaydisme et modernisation : émergence d'un nouvel universel politique?» Chroniques yéménites, $\mathrm{n}^{\circ} 13,2006$.

28 - Les Hachémites ou Banû Hâshim sont les descendants du prophète Mohammed par sa fille Fatima et son gendre 'Alî. Ils sont particulièrement révérés par toutes les tendances du chiisme, et occupent une place moins importante dans l'islam sunnite, majoritaire.

29 - Pour cette raison, on a pu aussi parler à leur endroit de «caste». 
peut-on «être zaydite en l'absence d'un imam ${ }^{30}$ »? En d'autres termes, le zaydisme existe-t-il en tant que communauté culturelle hors de cette théorie du pouvoir?

L'élite des sayyid ayant été soudainement marginalisée par les évènements politiques du $\mathrm{Xx}^{e}$ siècle, d'autres groupes prirent le pouvoir, mais sans avoir la même légitimité historique. Je reviendrai plus loin sur la nature sociologique de ces nouvelles élites. Arrêtons-nous seulement pour l'instant à la rupture induite par le renversement de l'imamat et l'instauration d'un régime républicain en 1962. De cette expérience politique est né un esprit anti-royaliste, qui est formulé selon des idéaux familiers aux oreilles européennes - démocratie, liberté, égalité - et qui s'exprime par un très fort rejet de tout ce qui touche au règne des Imams, confinant au tabou : actuellement encore, avoir des contacts avec les gens de la famille du dernier imam, la famille Hamid al-Dîn, est considéré comme une trahison, et le seul fait de mentionner le règne honni des Imams pourrait passer pour une tentative de les réhabiliter ${ }^{31}$. Aussi pour les historiens yéménites, il est difficile d'aborder ces périodes de manière objective ${ }^{32}$.

À ce vide politique s'ajoute un vide intellectuel : toute tentative moderniste d'écrire l'histoire se heurte à l'omniprésence de l'historiographie imamite et zaydite, car pendant des siècles, c'étaient surtout des sayyid qui rapportaient les évènements par écrit, donc selon leur propre version. Beaucoup étaient même des historiens attitrés du Palais, par exemple al-Jarmûzî pendant le règne des Qasimites au XVIII ${ }^{\mathrm{e}}$ siècle $^{33}$. Aussi est-il extrêmement difficile pour les Yéménites d'aujourd'hui d'écrire leur histoire politique, car les nouvelles élites se trouvent devant un vide culturel : peu de témoignages alternatifs, et aussi l'absence d'une période de l'histoire qui soit suffisamment valorisante ou à laquelle ils puissent se référer - du moins de leur point de vue, c'est-à-dire d'une manière emblématique et dans une optique identitaire.

Si les Yéménites perçoivent si mal leur histoire, c'est qu'ils semblent souvent confondre dans un même mouvement de rejet l'ensemble des différentes dynasties zaydites qui ont régné sur le Yémen pendant près de mille ans. Or celles-ci sont relativement nombreuses, et leur bilan peut faire l'objet d'appréciations très diverses. Pour ne prendre que les dernières, ne couvrant que l'histoire moderne, on peut citer :

30 - Gabriella Vom Bruck, «Being a Zaydi in the absence of an Imam?» in Rémy Leveau, Franck Mermier et Udo Steinbach (dir.), Le Yémen contemporain, Paris, Karthala, 1999, p. 169-192.

31 - Des contacts ont été établis entre le pouvoir républicain et la famille de l'imam Ahmed après l'Unité, mais beaucoup de contentieux patrimoniaux restent en suspens : par exemple le palais de l'imam Yahya, Dâr al-Khayr, est laissé délibérément à l'abandon. Il faut cependant noter un début de reconnaissance publique de cette histoire : la restauration du palais d'été de l'imam à Wâdî Dahr offre une représentation muséale relativement objective de la vie quotidienne de l'ancienne famille royale à l'intention d'un public populaire yéménite comme d'un public étranger.

32 - Lorsqu'en 2005, François Burgat publia un livre d'archives photographiques sur l'histoire du Yémen au cours du Xxe siècle, ce qui incluait nécessairement le règne des imams Yahya et Ahmed, il le fit contre l'avis de nombreux collègues yéménites. Cependant, après sa parution, le livre rencontra un vif succès auprès du public yéménite, soulagé de voir que la question avait été traitée aussi objectivement que possible.

33 - François Blukacz, «Le Yémen sous l'autorité des imams zaydites au XVII ${ }^{e}$ siècle : une éphémère unité», Revue des mondes musulmans et de la Méditerranée, n 67 : Le Yémen, passé et présent de l'unité, 1993. 
- la dynastie des Sharafeddine, au Xvi ${ }^{e}$ siècle, qui lutta farouchement contre l'occupation ottomane. N'ayant pas pu s'imposer définitivement en fondant un État, elle est entourée d'une auréole de résistance héroïque, donc d'une connotation moins négative que les autres;

- la dynastie des Qasimites, qui prit le pouvoir en 1610, et s'affirma en chassant les Turcs. Se maintenant au pouvoir pendant plus de deux siècles, fait exceptionnel au Yémen, ce fut une dynastie brillante qui laissa de nombreux édifices religieux, et aussi l'image d'un pouvoir fort. On connaît moins bien sa contribution au développement de la culture du café et à son exportation;

- les Hamid al-Dîn, qui reprirent le flambeau anti-ottoman lors de la deuxième occupation turque du Yémen (avec un embryon d'État dès 1904) et régnèrent officiellement sur le Yémen de 1919 à 1962.

Parmi ces trois dynasties, la troisième est bien sûr celle que les Yéménites connaissent le mieux. Mais bien que l'on puisse porter à son crédit d'avoir chassé les Turcs en 1919, cette dynastie fit subir au Yémen un régime tellement isolationniste et archaïque jusqu'en 1962, qu'elle suscita elle-même en grande partie le sentiment républicain qui allait mener à sa perte (même si ce sentiment doit aussi beaucoup à l'influence nassérienne dans les années 1950 et 1960).

Du règne des Imams, les Yéménites retiennent donc avant tout ces quarante et quelques années de despotisme particulièrement sombres, qui leur font négliger les neuf siècles précédents, comme un arbre qui leur cacherait la forêt ${ }^{34}$. Cette confusion peut être illustrée par une anecdote : en 2005, un historien yéménite reçut le soutien du Centre français d'archéologie et de sciences sociales pour publier des inventaires après décès des cinq derniers imams de la dynastie qasimite ${ }^{35}$ (donc l'avant-dernière), des documents qui couvrent une période allant d'environ 1750 à 1860 et présentent un intérêt certain pour l'histoire économique de la région de Sanaa, et plus particulièrement pour étudier le mode de transmission de ce patrimoine sur cinq générations ${ }^{36}$. Parallèlement au succès d'estime reçu par le livre de la part des rares spécialistes qui pouvaient en comprendre la valeur, il se trouva un journaliste yéménite pour accuser le Centre de «travailler pour le lobby de l'imam ». Plus révélatrice encore (tout en étant moins spectaculaire) fut la réaction de quelques hauts responsables de la culture et de la recherche au Yémen : sincèrement déçus et navrés pour les éditeurs, ils regrettaient que nous ayons «perdu notre temps avec des matériaux aussi peu intéressants »...

Dans cette situation trouble, il est particulièrement difficile de comprendre dans quelle mesure cette confusion entre différentes dynasties tient de la simple ignorance, et dans quelle mesure elle provient de partis pris politiques contemporains. On se

34 - Cela ne veut pas dire qu'il n'y avait pas de despotisme auparavant, mais l'histoire longue ne peut évidemment être réduite à ce seul aspect.

35 - Husayn al-Amrî (éd.), Le patrimoine des qasimites : cinq actes relatifs aux biens des imams du Yémen (1161-1288/1748-1871), Sanaa, Centre français d'archéologie et de sciences sociales de Sanaa/ Damas-Dâr al-Fikr, 2005 (en arabe).

36 - Ces documents montrent à la fois la richesse et le niveau de raffinement matériel de ces souverains, et détaillent particulièrement les propriétés immobilières de la famille. 
trouve peut-être dans une situation similaire à celle qui précéda l'épanouissement de la production historique en France au $\mathrm{xIx}^{\mathrm{e}}$ siècle $^{37}$. On peut cependant tenter de faire la part des choses à travers quelques exemples de réception de travaux historiques contemporains. Un événement historiographique particulier devait nous placer au coeur de cette problématique intellectuelle propre à l'intelligentsia yéménite actuelle.

\section{L'œuvre d'un historien du $x^{e}$ siècle, un enjeu politique contemporain}

Il y a au Yémen un historien et géographe majeur du $\mathrm{x}^{\mathrm{e}}$ siècle, al-Hamdânî, qui a laissé une Description de la péninsule Arabique et aussi une somme sur l'histoire du Yémen intitulée Le livre des couronnes (al-Iklîl). Le grand intérêt des ouvrages de Hamdânî, du moins de ceux que nous connaissons, c'est que, cherchant à donner une filiation ancienne aux Arabes du Sud, il se réfère à des généalogies antiques qui étaient encore conservées de manière orale à son époque, et qui sont par ailleurs assez bien connues des archéologues et des épigraphistes de l'Antiquité tardive. Du vivant de son auteur déjà, l'œuvre de Hamdânî avait soulevé de nombreuses controverses; elle lui avait même valu de faire de la prison pour avoir pris position contre les imams qui étaient en train d'asseoir leur pouvoir au Yémen ${ }^{38}$. L'un des axes polémiques de ces ouvrages, et de nombreux autres qui suivront dans la même veine, est le rejet de la domination généalogique des Hachémites, définis comme descendants de 'Adnân, ou Arabes du Nord (donc non yéménites), en leur opposant toute une généalogie parallèle, celle de Qahtân, qui serait spécifique de l'Arabie du Sud (donc du Yémen). Cette division segmentaire, qui apparut avant l'islam, fut régulièrement exploitée dans les conflits politiques du vIII ${ }^{\mathrm{e}}$ au $\mathrm{X}^{\mathrm{e}}$ siècle $^{39}$.

Le livre des couronnes est supposé comporter dix tomes, mais quatre seulement d'entre eux nous sont parvenus, les 1, 2, 8 et 10. Les autres ont-ils jamais existé? On ne pourrait l'assurer, car il est possible que cette idée des dix tomes ait été élaborée par des compilateurs plus tardifs ${ }^{40}$. Mais il n'est pas absurde de penser que certaines de ces œuvres aient été détruites par les Imams, leurs ennemis jurés... En tout état de cause, il y a un intérêt croissant des nouvelles élites pour cet auteur, dont les œuvres historiques avaient commencé à être éditées par les Yéménites dès

37 - Les années qui suivirent la Révolution française et la liquidation de la monarchie furent marquées par un régime républicain (ou bonapartiste) soucieux de rejeter toute trace de l'Ancien Régime. Cette tendance était inhérente à la mentalité révolutionnaire, par nature "a-historique» parce qu'elle "exalte le caractère inaugural du présent». En revanche, à la génération suivante, qui n'avait pas vécu la Révolution française, on vit une floraison extraordinaire d’historiens apportant des éclairages différents, souvent contradictoires, mais témoignant d'un débat fructueux (André Burguière, "Thiers, Tocqueville, Lamartine, Guizot... Le temps des historiens», Le Nouvel Observateur. Spécial XIX ${ }^{e}$. Le siècle de tous les possibles, $\mathrm{n}^{\circ} 2250 / 2251,2007-2008$, p. 97-98).

38 - Yusuf 'Abdallah, Al-Hamdânî, «la langue du Yémen». Études à l'occasion de son millénaire, Université de Sanaa, 1986 (en arabe et en anglais).

39 - Christian Robin, «L'Arabie antique de Karib'îl à Mahomet. Nouvelles données sur l'histoire des Arabes grâce aux inscriptions ", Revue des mondes musulmans et de la Méditerranée, n 61, 1991, p. 63-64.

40 - Notamment Nashwân Ben Sa'id al-Himyarî et son fils Mohammed, auteurs ayant vécu une centaine d'années après Hamdânî, et qui avaient eux aussi pris des positions anti-hachémites, sous la bannière qahtanite. 
les années $1970^{41}$. De leur côté, les plus hautes autorités de l'État avaient annoncé publiquement qu'elles offriraient une somme considérable à qui découvrirait un des tomes perdus du Livre des couronnes, ce qui, évidemment, aiguisa les appétits... À tel point que cette recherche allait donner lieu à un malentendu.

Il y a quelques années, un collectionneur yéménite proposa au Centre français d'archéologie et de sciences sociales de Sanaa d'éditer un manuscrit qu'il pensait être l'un des tomes manquants, le tome3, intitulé "La gloire de Qahtân et du Yémen". Comme on pouvait l'attendre d'un livre d'al-Hamdânî, la descendance de Qahtân, l'ancêtre éponyme des Arabes du Sud, c'est-à-dire des Yéménites, y était célébrée au détriment de celle de 'Adnân, l'ancêtre éponyme des Arabes du Nord, donc l'ancêtre des Hachémites, venus de La Mekke. L'intérêt de ce manuscrit (que l'on connaissait jusque-là seulement par son titre) est double : il donne des généalogies remontant à l'époque pré-islamique, notamment les fameux «huit ancêtres", al-mathâmina, du nom de huit tribus himyarites, témoignage précieux du passage de l'Antiquité à l'islam et moyen inestimable pour les archéologues de recouper leurs sources; mais c'est aussi un livre polémique du $\mathrm{x}^{e}$ siècle, pouvant rendre compte des conflits de l'époque et des termes dans lesquels ils étaient conduits.

Sur cette copie datant du XIX ${ }^{e}$ siècle (donc récente), une mention figurant au début du manuscrit indiquait qu'il s'agissait du "tome trois d'al-Iklîl ». Nous saisîmes cette occasion et commençâmes l'établissement du texte. Cependant, au fur et à mesure du travail, il parut de plus en plus douteux que l'œuvre puisse dater du $x^{e}$ siècle, et encore plus qu'elle puisse être d'al-Hamdânî. Par chance, nous trouvâmes dans une bibliothèque à l'étranger une autre copie plus ancienne et plus complète, et il s'avéra que c'était un ouvrage beaucoup plus tardif, composé au XIV siècle. Le titre n'était pas tout à fait identique à celui du tome 3 perdu («Les nouvelles de Qahtân et du Yémen"). Il ne s'agissait donc pas d'un tome du Livre des couronnes ${ }^{42}$. Même si, pour les historiens, l'ouvrage n'en était pas moins intéressant, il n'était plus possible de le publier avec ce titre et cette attribution d'auteur. Cependant, certains de nos collègues yéménites continuaient à vouloir croire qu'il s'agissait là du troisième tome tant recherché du Livre des couronnes, et ils le déclarèrent publiquement. Bien sûr, nous fûmes contraints d'apporter un ferme démenti ${ }^{43} \ldots$

Il faut replacer l'engouement soudain pour l'œuvre de Hamdânî, historien important pour l'identité du Yémen, dans le contexte politique récent. Depuis 2004, une révolte d'un mouvement zaydite fondamentaliste, le mouvement des Jeunes croyants, et sa violente répression, ont ensanglanté la région de Saada, dans le nord

41 - En premier lieu par Mohammed 'Alî al-Akwa' à Bagdad en 1977.

42 - La mention "tome trois d'al-Iklîl» ne figurait pas sur cette autre copie. Cette mention avait donc été rajoutée sur le manuscrit du collectionneur privé, probablement par le copiste du XIx ${ }^{e}$ siècle.

43 - Mounir Arbach et Mohammed Jazem, «Le livre Al-Fâsil bayn al-haqq wa-l-bâtil n'est pas le tome III d'al-Iklîl», Thawâbit, février 2008 (en arabe). Traduction en français : "Nouvelles données sur les Mafâkhir Qahtân wa-l-Yaman", Chroniques du manuscrit au Yémen, 5, en ligne : http://www.cefas. com.ye/cmy/no5_fev08/CMY5. 
du Yémen. Cette révolte a une connotation pro-hachémite très forte $e^{44}$. Comme mentionné plus haut, la région de Saada avait été la première où s'étaient installés les Imams en 898, et elle était toujours restée l'ultime bastion des imams zaydites ${ }^{45}$. Or, dans le cadre de la campagne sécuritaire et idéologique contre le mouvement des Jeunes croyants, il est évident que la découverte du manuscrit d'un tome inédit d'al-Iklîl, surtout le tome 3 dont la rumeur disait qu'il menait une polémique contre les Hachémites, aurait été du pain béni pour les nécessités de la propagande antisayyid: elle lui aurait apporté une légitimité historique prestigieuse ${ }^{46}$.

\section{Sociologie des élites politiques actuelles}

À la faveur du renversement des Imams et de la marginalisation des Hachémites, deux groupes sociaux ont conquis l'essentiel du pouvoir : les chefs de tribus et les «juges».

Les tribus, en particulier certains clans de la confédération des Hâshed (qui avait toujours été le fer de lance militaire des Imams), contrôlent désormais l'armée et le pouvoir suprême, selon une alliance complexe entre militaires originaires de cette tribu et certains sheykh importants. À côté de cette alliance stratégique, on trouve la classe sociale des "juges", les qâdî, qui fournissaient sous les Imams une grande partie des hauts fonctionnaires et intermédiaires, ainsi que des cadres religieux, mais qui ne pouvaient en aucun cas accéder au pouvoir suprême, puisqu'ils n'étaient pas hachémites $^{47}$; leur essor date surtout de la période qasimite ${ }^{48}$. À la faveur de la Révolution et de la chute des sayyid, ce groupe a bénéficié du vide qui s'est créé dans toutes les fonctions administratives requérant une bonne formation intellectuelle. C'est ainsi que cette classe a fourni au Yémen une grande partie de son personnel politique, dont le deuxième président de la République, le qâdî Abd al-Rahman alIryanî, et le troisième président, Ibrahîm al-Hamdî, ainsi que de nombreux ministres dans tous les gouvernements successifs, et d'innombrables hauts fonctionnaires.

Quels que soient les caractéristiques et les aléas de l'alliance actuelle entre ces deux groupes, alliance qui dure depuis au moins la Révolution de 1962, on ne peut que constater que ce sont des membres de ces deux groupes qui sont précisément à la

44 - Même si elle n'a semble-t-il jamais prétendu restaurer l'imamat, comme l'en accusent ses détracteurs. Voir Samy Dorlian, "L'identité hachémite à l'épreuve de la guerre de Saada", Chroniques Yéménites, $\mathrm{n}^{\circ} 15$, sous presse.

45 - La région de Saada n'a jamais été totalement intégrée à la République, car elle n'avait pas été conquise lors de la guerre civile, mais simplement pacifiée à la suite d'un traité de paix en 1970. Les sayyid y entretiennent encore des relations privilégiées, spirituelles et politiques, avec les tribus locales, ce qui explique sans doute en partie que ce conflit ait duré depuis quatre ans (Samy Dorlian, art. cit., 2008).

46 - Dans le même esprit, cette propagande était élaborée dans un «centre de recherche» baptisé par ses promoteurs "Centre Nashwân Ben Sa'îd al-Himyarî», du nom même de ce partisan d'alHamdânî du XI e siècle cité plus haut (S. Dorlian, art. cit., 2008).

47 - À l'époque des Imams, les qâdî et les sayyid étaient cependant réunis en tant qu'hommes de religion, sous une même appellation, «le drapeau blanc» ('alem al-bayâd), allusion à leur robe et à sa couleur symbole de pureté rituelle.

48 - Muhammad Sbitlî, "La société et l'État vus par les qâdî», Hawliyyât yamaniyya, n 3, 2006, p. 137 (en arabe). 
recherche de matériaux historiques pouvant servir à une construction identitaire nationale qui se passerait de la référence hachémite. Cette tentative n'est pas aisée, car ces intellectuels et ces hommes politiques sont également pris dans une problématique identitaire liée à leur histoire et à leur appartenance communautaire, qu'elle soit réelle ou imaginée : étant historiquement zaydites, mais s'étant souvent affranchis politiquement du zaydisme imamite, ils balancent à l'évidence entre une rupture totale avec le zaydisme et l'invention d'un zaydisme sans imam (où l'imam est de fait le président de la République).

Curieusement, la première tendance avait commencé assez tôt, à la fin du XVIII ${ }^{\mathrm{e}}$ siècle : l'imam al-Shawkânî ${ }^{49}$, un savant d'origine zaydite, avait tenté d'édulcorer les aspects sectaires du zaydisme dans le domaine des sciences religieuses. À la suite du succès du royaume qasimite qui avait conquis une grande partie du Yémen actuel ${ }^{50}$, les imams zaydites dominaient le pays, mais ils s'étaient retrouvés en minorité dans un pays majoritairement shafiite, et donc sunnite ${ }^{51}$; pour faire face à cette situation, alShawkânî avait cherché à établir une voie moyenne entre les deux écoles, répondant à une volonté pragmatique des souverains de l'époque d'homogénéiser le Yémen ${ }^{52}$ sur une base que l'on pourrait qualifier dès cette période de "nationale». En faisant plusieurs pas vers le sunnisme, Shawkânî finit par lui être assimilé; c'est du moins la perception qu'en ont ses descendants spirituels, ainsi que ceux qui les ont étudiés. Il faut souligner l'importance symbolique et identitaire de cette école de pensée qui a laissé des traces importantes dans le «zaydisme» contemporain, notamment dans la classe des qâdî (à laquelle appartenait Shawkânî), ceux-là même qui dominèrent les débuts de la République ${ }^{53}$. Les historiens qui ont promu al-Hamdânî à l'époque contemporaine appartiennent également à cette mouvance.

Une seconde tendance intellectuelle consiste, tout en rejetant la théorie politique du zaydisme, à essayer de sauver ce qui peut encore l'être de l'héritage intellectuel zaydite, notamment en insistant sur son rationalisme: certains historiens mettent en valeur le mouvement de la Mutarrafiyya, un courant du zaydisme qui, au Moyen Âge, avait essayé de se passer des sayyid (en ne posant pas l'appartenance à la famille hachémite comme une condition indispensable pour diriger la communauté). Ce mouvement avait été exterminé au XII ${ }^{\mathrm{e}}$ siècle par l'imam Abdallah Bin Hamza et ne fut redécouvert que dans les années 1990 par quelques penseurs zaydites, et aussi par des marxistes ${ }^{54}$. Une troisième tendance, qui ne se distingue pas clairement de la seconde, cherche à démontrer qu'il exista historiquement au Yémen un zaydisme avant l'imam al-Hâdî, le fondateur du IX siècle. On prétend alors que le zaydisme

49 - Et plusieurs autres comme Ibn al-Amîr et al-Muqbilî.

50 - François Blukacz, "Le Yémen sous l'autorité des imams zaydites au XVII esiècle...», art. cit., 1993, p. 48-49.

51 - L'école shafiite, d'obédience sunnite, tire son nom du théologien Abu Abdallah Muhammad ibn Idris al-Shafi'I (767-820), dont l'apport à la législation musulmane a été considérable. (NDLR)

52 - Bernard Haykel, "Shawkânî and the Jurisprudential Unity of Yemen», Revue des mondes musulmans et de la Méditerranée, $\mathrm{n}^{\circ} 67$ : Le Yémen, passé et présent de l'unité, 1993, p. 55.

53 - Bernard Haykel, art. cit., 1993, p. 63.

54 - Alî Muhammad Zayd, Les tendances de la pensée mo'tazilite au Yémen au VI-XII siècle, Sanaa, CFEY, 1997 (en arabe). 
yéménite aurait été dévoyé dès al-Hâdî, ce qui est appelé le "hadâwisme» ${ }^{55}$, alors étiqueté comme allogène (voir note 23). Mais cette hypothèse reste à démontrer sur le plan historique puisque, dans l'état actuel des sources disponibles, on ne connaît pas de penseur ou de militant zaydite spécifiquement yéménite avant 898 . Toutes ces tentatives partent à l'évidence du désir de trouver un fil historique permettant d'attester une continuité du zaydisme hors des Hachémites, donc de "yéméniser» le zaydisme, selon une démarche très contemporaine et rétroactive.

Si des intellectuels et hommes politiques zaydites "historiques » ou " statistiques $^{56}$ » ont recherché des bases théologiques à cet élargissement de l'identité zaydite dès le XVIII $^{\mathrm{e}}$ siècle, il est intéressant de se demander si les intellectuels qui seraient aujourd'hui entièrement "dé-zaydisés » ou complètement "sunnisés » vont jusqu'à rechercher des références non zaydites dans l'histoire de leur pays. Ils pourraient, par exemple, le faire avec le royaume rasoulide, majoritairement sunnite, qui fut fondé entre le XIII ${ }^{\mathrm{e}}$ et le XIv ${ }^{\mathrm{e}}$ siècle par la dynastie la plus florissante que le Yémen ait jamais connue. Mais il ne semble pas que ce soit le cas : très peu de ces historiens ou pseudo-historiens s'y intéressèrent ${ }^{57}$. Peut-être le manque d'intérêt pour cette dynastie tient-il au fait qu'elle était d'origine kurde, et qu'elle se forgea une généalogie arabe ${ }^{58}$, des Ghassanides (c'est-à-dire des Arabes du Nord), sans que, d'ailleurs, cela convainque personne, et d'autant moins dans le contexte nationaliste actuel. En revanche, cette dynastie très ancienne suscite beaucoup plus d'intérêt chez les intellectuels chafiites, originaires des mêmes régions où ce royaume s'était épanoui, pour des motivations communautaires évidentes, et qui sont encore vivaces aujourd'hui; le fait que cette dynastie ait eu une origine étrangère est alors perçu plutôt comme un élément positif d'ouverture vers la Syrie et l'Égypte, phares de la civilisation islamique à cette époque.

Ces débats historiques, et bien d'autres du même type, posent plusieurs questions identitaires fondamentales : quelle peut-être la nature du zaydisme dans un Yémen où cette communauté, tout en restant dominante, est de plus en plus minoritaire, minée par de nombreuses dissensions, partagée par des courants intellectuels contradictoires, et surtout privée d'un leadership politique traditionnel ? Compte tenu de l'importance de la théorie du pouvoir dans l'identité du zaydisme yéménite, ce problème se pose de manière particulièrement aiguë. Par ailleurs, comment construire l'identité contemporaine du Yémen sans la composante culturelle du zaydisme et son patrimoine intellectuel si original et si marquant ${ }^{59}$ ? La seule alternative est-elle une sunnisation totale qui pourrait s'opérer sur le mode panislamique (du type

55 - Abdallah al-Shamâhî, The Biography of Imam al-Mansûr Abdallah ibn Hamza (d. 1217), a Critical Study, Phd, université de Glasgow, 2004

56 - Au sens où l'on parlait des «musulmans statistiques» dans les ex-républiques de l'URSS, pour désigner ceux qui, musulmans par leur origine et leur état civil, étaient sensés ne pas être pratiquants.

57 - À l'exception notable du vénérable qâdî Ismâ'îl al-Akwa', historiographe de haute stature et représentant éminent du «zaydisme dé-zaydisé». Voir son petit ouvrage L'État rasoulide (2004), édité à Aden.

58 - Tentative dont le manuscrit «Les nouvelles de Qahtân et du Yémen» témoigne justement.

59 - Le zaydisme conserve par inertie un patrimoine culturel important : rituels religieux et traditions populaires, où figurent notamment la mention privilégiée de la famille du Prophète dans les prières et la poésie chantée dans les cérémonies de mariage et de toutes les autres occasions du cycle de la vie. 
du parti Islâh, le Parti yéménite pour la réforme) ? Ou bien une «arabisation » sur un mode nationaliste ${ }^{60}$ ? Les nouvelles élites, tribales et «juges», seront-elles capables de redonner à cette communauté une cohésion idéologique lui permettant de continuer à exister politiquement dans l'avenir? Ou bien au contraire, le regard critique, pour ne pas dire déconstructeur, qu'elles portent sur l'histoire du Yémen minera-t-il le ciment qui a fait que cette communauté a pu présider aux destinées du pays pendant des siècles? Ces questions vont bien au-delà du politique, et conditionnent à terme la configuration identitaire du Yémen de l'avenir.

C'est donc bien dans le contexte d'une tentative consciente de construire une identité nationale en fonction des coordonnées propres de la structure sociopolitique du Yémen contemporain qu'apparaît la nécessité de faire pièce au patrimoine intellectuel hachémite, surdimensionné au Yémen. C'est sans doute cette nécessité qui explique l'ouverture vers l'Antiquité, dans les manuels d'histoire et les ouvrages de vulgarisation à destination du grand public. Comme nous l'avons vu, al-Hamdânî, cet historien et géographe ancien, permettait dès le $\mathrm{x}^{e}$ siècle $\mathrm{d}^{\prime}$ ouvrir cette porte vers un modèle légendaire alternatif à un islam venu du nord. Et il a transmis aux élites contemporaines une filiation intellectuelle, si ce n'est généalogique, d'une histoire du Yémen qui ne soit pas hachémite.

Si l'on transpose la problématique que Prasenjit Duara a appliquée à la Chine, on pourrait dire qu'à l'écriture d'une histoire dominante succède, non pas «l'écriture d'une histoire des opprimés", mais celle d'une nouvelle histoire dominante, qui reste cependant en chantier.

Dans un contexte yéménite où la tentation essentialiste, qu'elle soit intégriste ou chauvine, est très prégnante, cette diversité des références historiques identitaires est peut-être un gage de pluralisme : même si elles ne sont pas toujours nuancées, ces références divergentes sont sources de débat et de réflexion, chez les intellectuels et dans une large portion de la population. Mais il faudra encore sans doute une longue élaboration scientifique et intellectuelle (avec ou sans les chercheurs étrangers) pour que les constructions identitaires yéménites acceptent de faire se rencontrer les différents imaginaires sociaux avec une version consensuelle des faits historiques.

60 - Ces possibilités intellectuelles sont chacune influencées par deux tendances politiques plus globales, l'une, islamique, et l'autre, nationaliste arabe. Poussée dans son extrême logique, la tendance panislamique ne pourrait aboutir qu'à une dissolution totale de l'identité zaydite. En revanche, la tendance nationalise arabe, particulièrement dans sa version baathiste, peut permettre d'opérer une sorte de "sémitisation» et simultanément d'«arabisation» de l'histoire du Yémen, valorisant les relations entre les anciens rois du Yémen et ceux du croissant fertile antique. D'après ces nouvelles "légendes urbaines», la ville d'Ougarit en Syrie aurait été fondée par des Yéménites (Farah, Les tubbấ' sabéens du Yémen, op. cit., 2002, p. 39) et les Araméens tireraient leurs origines d'une conquête de la Mésopotamie au $\mathrm{Xv}^{e}$ siècle avant J.-C. par des Yéménites de la ville d'Iram (seulement connue par le Coran, donc plus tardive) (ibid., p. 41-43, 44). Cette relation ancienne entre le Yémen d'une part et la Syrie et l'Irak d'autre part, élaborée dès les années 1980, fait clairement écho aux bonnes relations du Yémen avec les deux pays où est né le parti nationaliste Baath contemporain, en particulier avec l'Irak de Saddam Hussein. L'avantage d'une telle version est d'être compatible avec le nationalisme yéménite, qui devient en quelque sorte une version sudarabique du baathisme, et de ne pas remettre en cause les aspects culturels, dépolitisés, du zaydisme par ailleurs valorisé pour son rationalisme. 


\section{Bibliographie}

- 'AbDallah Y., Al-Hamdânî, "la langue du Yémen». Études à l'occasion de son millénaire, Université de Sanaa, 1986 (en arabe et en anglais).

- AкwA' Ismâ 'îl b. 'Alî, L'État rasoulide au Yémen (1228-1454), Aden, Université d'Aden, 2003 (en arabe).

- 'AMrî Husayn al-, Le patrimoine des qasimites : cinq actes relatifs aux biens des imams du Yémen (1161-1288/1748-1871), Sanaa et Damas, CEFAS / Dâr al-Fikr, 2005 (en arabe).

- Arbach M. et R. Crassard, "L'Arabie du Sud vue de l'intérieur. Recherches archéologiques menées par les Yéménites», Chroniques yéménites, $\mathrm{n}^{\circ} 14,2007, \mathrm{p} .1-14$ (http:// cy.revues.org/).

- Arbach M. et M. JAZîM «Le livre Al-Fâsil bayn al-haqq wa-l-bâtil n'est pas le tome III d'alIklîl», Thawâbit, février 2008 (en arabe). Traduction en français : "Nouvelles données sur les Mafâkhir Qahtân wa-l-Yaman", Chroniques du manuscrit au Yémen, 5 (en ligne, http:// www.cefas.com.ye/cmy/no5_fev08/CMY5).

- Beaucamp J., F. Briquel-Chatonnet et C. Robin, «La persécution des chrétiens de Najrân", ARAM, n 11-12, 1999-2000, p. 15-83 (paru en 2001).

- Beydoun A., Identité confessionnelle et temps social chez les historiens libanais contemporains, Beyrouth, Université libanaise, 1984.

- Blukacz F., «Le Yémen sous l'autorité des imams zaydites au $\mathrm{XVII}^{\mathrm{e}}$ siècle: une éphémère unité», Revue des mondes musulmans et de la Méditerranée, $\mathrm{n}^{\circ} 67$ : Le Yémen, passé et présent de l'unité, 1993, p. 39-51.

- Burguière A., "Thiers, Tocqueville, Lamartine, Guizot... Le temps des historiens", Le Nouvel Observateur. Spécial XIX ${ }^{e}$ Le siècle de tous les possibles, $\mathrm{n}^{\circ} 2250 / 2251,2007-2008$, p. 97-98.

- Burgat F. (dir.), Le Yémen vers la République : iconographie historique du Yémen (19001970), Sanaa, Centre français d'archéologie et de sciences sociales de Sanaa, 2005.

- CARApico S., «Arabia Incognita. An Invitation to Arabian Peninsula ", in M. al-RASHEED et R. Vitalis, Counter-narratives: History, Contemporary Society and Politics in Saudi Arabia and Yemen, New York, Palgrave Macmillan, 2003.

- Dorlian S., "Zaydisme et modernisation : émergence d'un nouvel universel politique?» Chroniques yéménites, $\mathrm{n}^{\circ} 13,2006$.

- Dorlian, S., «L'identité hachémite à l'épreuve de la guerre de Saada", Chroniques Yéménites, $\mathrm{n}^{\circ} 15$, sous presse.

- Duara Prasenjit, Rescuing History from the Nation: Questioning Narratives of Modern China, Chicago, University of Chicago Press, 1995.

- Farah M. al-, Les tubbâ' sabéens du Yémen, Sharja, Dâr al-Thaqâfa al-'Arabiyya, 2002 (en arabe).

- FARAh M. al-, Découvertes récentes sur l'histoire de l'État et de la civilisation de Sabâ, Sanaa, Ministère de la Culture, 2004 (en arabe).

- Gochenhour, «Towards a Sociology of the Islamization of Yemen", in B. R. PrIDHAM, Contemporary Yemen, Politics and Historical Background, Londres, Croom Helm, University of Exeter, 1984.

- HaddÂD M. Yahyâ al-, Histoire générale du Yémen. I. Avant l'islam; II. Dans le cortège de l'islam; III. Le Yémen moderne et contemporain, Sanaa, Ministère de la Culture et du Tourisme, 2004 (en arabe).

- Haykel B., "Shawkânî and the Jurisprudential Unity of Yemen", Revue des mondes musulmans et de la Méditerranée, $\mathrm{n}^{\circ} 67$ : Le Yémen, passé et présent de l'unité, 1993.

- Haykel B., Revival and Reform in Islam: The Legacy of Muhammad al-Shawkani, Cambridge/New York, Cambridge University Press, 2003.

- Lambert J., «Sabâ et le barrage de Mareb. Récit fondateur et temps imaginé», Peuples de la Méditerranée, $\mathrm{n}^{\circ}$ 56-57, juilletdécembre 1991, p. 141-176.

- Mermier F., «Les héritages d'une histoire morcelée», in R. LEVEAU, F. MERMIER et U. Steinbach (dir.), Le Yémen contemporain, Paris, Karthala, 1999, p. 6-35. 
- Mermier F., "Présentation", Revue des mondes musulmans et de la Méditerranée, $\mathrm{n}^{\circ} 121$ 122 : Yémen Territoires et identités, avril 2008, p. 9-16 (en ligne).

- Rasheed M. al- et R. Vitalis, Counternarratives: History, Contemporary Society and Politics in Saudi Arabia and Yemen, New-York, Palgrave Macmillan, 2003.

- Robin C., «L'Arabie antique de Karib'îl à Mahomet. Nouvelles données sur l'histoire des Arabes grâce aux inscriptions", Revue des mondes musulmans et de la Méditerranée, $\mathrm{n}^{\circ} 61,1991$.

- Sbitlî M., "Pouvoir de la loi religieuse et légitimité du pouvoir. Les relations entre les qâdî et l'État dans l'histoire du Yémen moderne", in C. Robin, M. JAZîm (dir.), Le pèlerin des forteresses du savoir. Hommage au qâdî Ismâ'îl b. 'Alî al-Akwa', Sanaa, Centre français d'archéologie et de sciences sociales de Sanaa, 2006, p. 287-331 (en arabe).
- Sbitlî M., «La société et l'État vus par les qâdî», Hawliyyât yamaniyya, n 3, 2006, p. 135-157 (en arabe).

- ShamÂHî, A. al-, The Biography of Imam alMansûr Abdallah ibn Hamza (d. 1217), a Critical Study, Phd, université de Glasgow, 2004.

- Tuchscherer M., "Introduction», Revue des mondes musulmans et de la Méditerranée, $\mathrm{n}^{\circ} 67$ : Le Yémen, passé et présent de l'unité, 1993.

- Vom Bruск G., « Being a Zaydi in the Absence of an Imam?» in R. LEVEAU, F. MERMIER et U. Steinbach (dir.), Le Yémen contemporain, Paris, Karthala, 1999, p. 169-192.

- Yémen, territoire et identité, P. Chevalier, V. Martignon et J. Schiettecatte (dir.), Revue des Mondes musulmans et de la Méditerannée, $\mathrm{n}^{\circ}$ 121-122, avril 2008 .

- ZAYD Alî Muhammad, Les tendances de la pensée mo'tazilite au Yémen au VI-XII siècle, Sanaa, CFEY, 1997 (en arabe). 